\title{
Does the Fuhrman or World Health Organization/ International Society of Urological Pathology Grading System Apply to the Xp11.2 Translocation Renal Cell Carcinoma?
}

\section{A 10-Year Single-Center Study}

\author{
Ning Liu, ${ }^{*}$ Weidong Gan, ${ }^{*}$ Feng Qu, ${ }^{*}$ Zhen Wang, ${ }^{*}$ Wenyuan Zhuang, ${ }^{*}$ Sezim Agizamhan, ${ }^{*}$ Linfeng Xu, Juanjuan Yin, \\ Hongqian Guo, ${ }^{*}$ and Dongmei $\mathrm{Li}^{\ddagger}$
}

\begin{abstract}
From the Department of Urology, ${ }^{*}$ Nanjing Drum Tower Hospital, The Affiliated Hospital of Nanjing University Medical School, Nanjing, China; the Laboratory of Genitourinary Cancer Pathogenesis, ${ }^{\dagger}$ National Cancer Institute, National Institutes of Health, Bethesda, Maryland; the Immunology and Reproduction Biology Laboratory \& State Key Laboratory of Analytical Chemistry for Life Science, ${ }^{\ddagger}$ and the Jiangsu Key Laboratory of Molecular Medicine, Medical School, Nanjing University, Nanjing, China
\end{abstract}

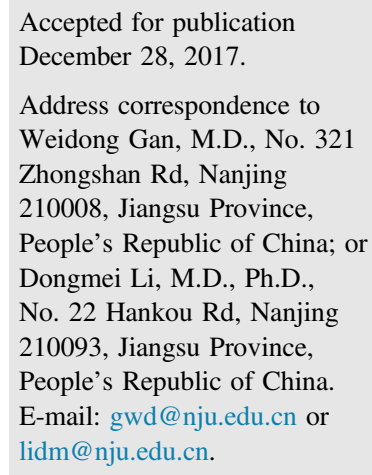

\begin{abstract}
The Fuhrman and World Health Organization/International Society of Urological Pathology (WHO/ISUP) grading systems are widely used to predict survival for patients with conventional renal cell carcinoma. To determine the validity of nuclear grading systems (both the Fuhrman and the WHO/ISUP) and the individual components of the Fuhrman grading system in predicting the prognosis of Xp11.2 translocation renal cell carcinoma (Xp11.2 tRCC), we identified and followed up 47 patients with Xp11.2 tRCC in our center from January 2007 to June 2017. The Fuhrman and WHO/ISUP grading was reassigned by two pathologists. Nuclear size and shape were determined for each case based on the greatest degree of nuclear pleomorphism using image analysis software. Univariate and multivariate analyses were performed to evaluate the capacity of the grading systems and nuclear parameters to predict overall survival and progression-free survival. On univariate Cox regression analysis, the parameters of nuclear size were associated significantly with overall survival and progression-free survival, whereas the grading systems and the parameters of nuclear shape failed to reach a significant correlation. On multivariate analysis, however, none of the parameters was associated independently with survival. Our findings indicate that neither the Fuhrman nor the WHO/ISUP grading system is applicable to Xp11.2 tRCC. The assessment of nuclear size instead may be novel outcome predictors for patients with Xp11.2 tRCC. (Am J Pathol 2018, 188: 929-936; https://doi.org/10.1016/j.ajpath.2017.12.018)
\end{abstract}

Xp11.2 translocation renal cell carcinoma (Xp11.2 tRCC), a rare subtype of RCC, is derived from several different chromosomal translocations of Xp11.2 breakpoints and the formation of transcription factors binding to the IGHM enhancer 3 (TFE3) fusion genes. Different from conventional RCC subtypes, Xp11.2 tRCC tends to advance to the late stages rapidly and manifest an aggressive and invasive clinical course. ${ }^{1,2}$ In our previous study, ${ }^{3}$ the tumor node metastases (TNM) stage and inferior vena cava tumor thrombosis were identified as independent prognostic factors by univariate and multivariate analyses. Although nuclear grading now widely is accepted as having prognostic significance, particularly in clear-cell RCC (CCRCC) and papillary RCC (PRCC), ${ }^{4}$ there

Supported by National Natural Science Foundation of China grant 81572512 (W.G.).

Disclosures: None declared. 
remains a lack of systematic evaluation of the prognostic value of nuclear grading in Xp11.2 tRCC.

Over the past 30 years, the four-tiered Fuhrman grading system, which evaluates nuclear size, nuclear shape, and the presence of nucleolar prominence simultaneously, has been the most widely used grading protocol all over the world. ${ }^{5}$ Despite its popularity, the Fuhrman grading system has some inherent drawbacks. The criteria for grading nucleolar prominence and nuclear shape are somewhat poorly defined in the classification. Moreover, the inter-relationship between these tedious components remains undetermined. If any discordance occurs, no rules are available for assigning a weighting to the grading system. These limitations are probably responsible for its poor to, at best, moderate reproducibility.

In 2012 the International Society of Urological Pathology (ISUP) Consensus Conference proposed a novel grading system, which was accepted and recommended for applying to CCRCC and PRCC by the World Health Organization (WHO) in 2016. ${ }^{7,8}$ In the WHO/ISUP grading system, the first three grades are defined based on nucleolar prominence. Grade 4 is defined by the presence of pronounced nuclear pleomorphism, tumor giant cells, and/or rhabdoid and/or sarcomatoid differentiation. A comparison of the Fuhrman and WHO/ISUP grading systems is shown in Table 1.

Compared with the conventional four-tiered Fuhrman grading system, findings based on CCRCC indicate that a simplified Fuhrman grading system produces equally valid conclusions and can reduce interobserver variance. ${ }^{9,10}$ The relevance of simplified Fuhrman grading systems for $\mathrm{Xp11.2} \mathrm{tRCC} \mathrm{is} \mathrm{unknown.} \mathrm{Therefore,} \mathrm{this} \mathrm{study} \mathrm{was} \mathrm{per-}$ formed to assess the prognostic significance of conventional/simplified Fuhrman and WHO/ISUP grading systems in a series of Xp11.2 tRCC patients, and to investigate the prognostic utility of tumor nuclear size alone or nuclear shape alone, as well as the inter-relationship between tumor nuclear size and nuclear shape.

\section{Materials and Methods}

\section{Study Population}

All procedures were approved by the Medical Ethics Committee for Human Experiments of Nanjing Drum Tower Hospital. Between January 2007 and June 2017, a total of 1478 RCC patients underwent partial nephrectomy or radical nephrectomy at Nanjing Drum Tower Hospital. Altogether, 115 cases showed positive immunohistochemical staining or microscopic features that included abundant clear or eosinophilic cytoplasm, irregular nuclei with vesicular chromatin, and prominent nucleoli with papillary, nested, alveolar, or tubular architectures. Polyclonal breakapart probes for TFE3 gene rearrangement at the Xp11.2 region were applied. Forty-seven of these 115 cases ultimately were identified as Xp11.2 tRCC. The detailed diagnostic techniques of Xp11.2 tRCC have been published by us previously. ${ }^{11}$ Details of patients' age, sex, maximum tumor diameter, TNM staging, and surgery procedures were collected and their follow-up evaluations were recorded.

\section{Research Methods}

All sections were reviewed by two dedicated pathologists (Jun Yang and Ming Chen, Nanjing Drum Tower Hospital, Nanjing, China). For each case, a high-power field that showed the greatest degree of nuclear pleomorphism was identified, based on which Fuhrman or WHO/ISUP grading was assigned. Of note, the assessment of the Fuhrman grading was subjective. If there was grading discordance between pathologists, the final grading was decided after discussion. The simplified two-tiered Fuhrman grading, proposed by Zisman et al, ${ }^{9}$ was stratified as low grade (grades I and II) and advanced grade (grades III and IV). The simplified three-tiered Fuhrman grading was stratified according to Ficarra et al, ${ }^{10}$ who proposed combining grades I and II, although grades III and IV were kept separate.

Parameters of nuclear size and shape were quantified by image analysis. Images were captured, magnified, and

Table 1 Comparison of Conventional Four-Tiered Fuhrman Grading and WH0/ISUP Grading System

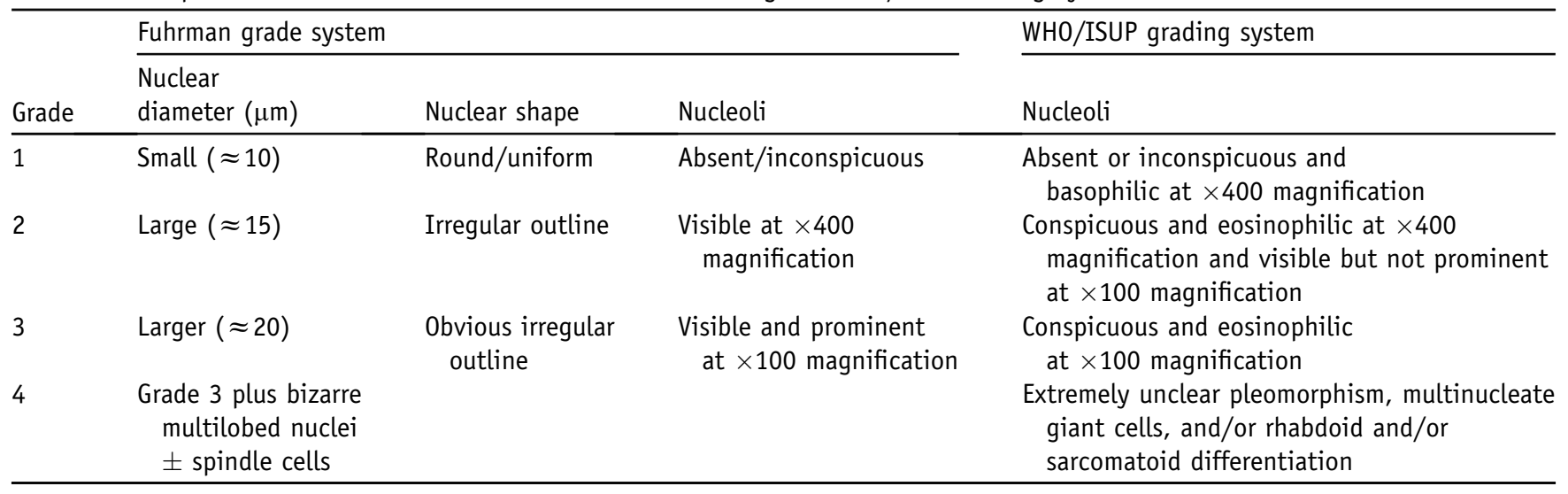

WHO/ISUP, World Health Organization/International Society of Urological Pathology. 
enhanced by an Olympus Provis BX43 research transmission light microscope. An Olympus DP27 microscope digital camera with a 5-megapixel resolution and a cellSens Entry controller software package version 1.12 (Olympus Corporation, Tokyo, Japan) were used for digital data recording and analysis. One hundred nuclei within the high-power field of each case were analyzed using the Image Pro Plus software version 6 (Media Cybernetics, Inc., Rockville, MD). Measurements of nuclear size were recorded as follows: i) the nuclear perimeter, ii) the nuclear area (the sum of calibrated pixel units found enclosed within specific boundaries), and iii) the length of the major nuclear axis. Measurements of nuclear size were recorded as the shape factor $(4 \pi$ area/ perimeter ${ }^{2}$ ) and nuclear compactness (perimeter ${ }^{2} /$ area).

TNM staging was categorized according to the Seventh American Joint Committee on Cancer staging criteria (2010). All of the patients were followed up every 3 months in the initial 2 years, every 6 months during the following 3 years, and annually after 5 years until the time of death or loss to follow-up evaluation. Both local recurrence and systemic metastasis were regarded as disease progression. Progressionfree survival (PFS) was defined as the time interval between the date of surgery and the date of disease progression or censoring at the time of last follow-up evaluation. Overall survival (OS) was defined from the initiation of surgery to the date of death or last follow-up evaluation.

\section{Statistical Analysis}

Continuous data are presented as a median (range) and categoric data are presented as a number (proportion). Oneway analysis of variance and the $t$-test were performed for statistical comparisons. Univariate and multivariate Cox regression was used to evaluate the predictive role of all parameters for survival. PFS and OS survival curves were obtained by Kaplan-Meier analysis. All statistical analyses were performed using SPSS software version 23.0 (SPSS, Inc., Chicago, IL), and figures were generated with GraphPad Prism software version 5.0 (GraphPad Software, San Diego, CA). $P$ values $<0.05$ were considered statistically significant.

\section{Results}

In total, 47 cases of Xp11.2 tRCC were identified, with an incidence of $3.2 \%$. Twenty (42.6\%) cases were male and 27 $(57.4 \%)$ cases were female, with a mean age of 31 years (range, 3 to 71 years). The maximum diameters of the tumors ranged from 3 to $13 \mathrm{~cm}$ with a mean dimension of $5.43 \mathrm{~cm}$. There was one case of distant metastasis at diagnosis for which the patient elected not to undergo surgery, the other 46 cases showed no sign of metastasis and underwent either partial nephrectomy (15 cases; $31.9 \%$ ) or radical nephrectomy (31 cases; 66.0\%). The characteristics of those 46 patients are shown in Table 2.

For the conventional four-tiered Fuhrman grading system, $1(2.1 \%)$ case was marked as grade $1,25(53.2 \%)$ cases
Table 2 Characteristics of 46 Cases of Xp11.2 tRCC Patients Treated with Either Radical or Partial Nephrectomy

\begin{tabular}{lc}
\hline Variable & $n(\%)$ \\
\hline Maximum diameter of tumor & \\
$4 \mathrm{~cm}$ & $21(45.7)$ \\
$>4 \mathrm{~cm}$ to $7 \mathrm{~cm}$ & $16(34.8)$ \\
$>7 \mathrm{~cm}$ to $10 \mathrm{~cm}$ & $7(15.2)$ \\
$>10 \mathrm{~cm}$ & $2(4.3)$ \\
R.N.E.A.L score & \\
$4-6$ & $16(34.8)$ \\
$7-9$ & $11(23.9)$ \\
$10-12$ & $19(41.3)$ \\
pTNM stage & \\
1 & $31(67.4)$ \\
2 & $4(8.7)$ \\
3 & $8(17.4)$ \\
4 & $4(8.7)$ \\
pT stage & \\
1 & $32(69.6)$ \\
2 & $4(8.7)$ \\
3 & $6(13.0)$ \\
4 & $4(8.7)$ \\
pN stage & \\
Nx & $24(52.2)$ \\
N0 & $14(30.4)$ \\
N1 & $8(17.4)$ \\
\hline
\end{tabular}

$\mathrm{pN}$, pathological node metastasis stage; $\mathrm{pT}$, pathological tumor invasion stage; pTNM, pathological TNM stage; R.N.E.A.L., radius, exophytic extent, nearness to the renal sinus, anterior/posterior location, location relative to the polar lines; tRCC, translocation renal cell carcinoma.

were marked as grade 2, $18(38.3 \%)$ cases were marked as grade 3 , and $3(6.4 \%)$ cases were marked as grade 4 . Grades I and II together reached 55.3\%, and grades III and IV accounted for $44.7 \%$. Division of cases based on WHO/ ISUP grading resulted in grade 1 for $1(5.3 \%)$ cases, grade 2 for $12(26.3 \%)$ cases, grade 3 for $29(61.7 \%)$ cases, and grade 4 for $4(8.5 \%)$ cases. The distribution of each of the WHO/ISUP grading tiers within the Fuhrman grading outcome is shown in Figure 1.

WHO/ISUP grade

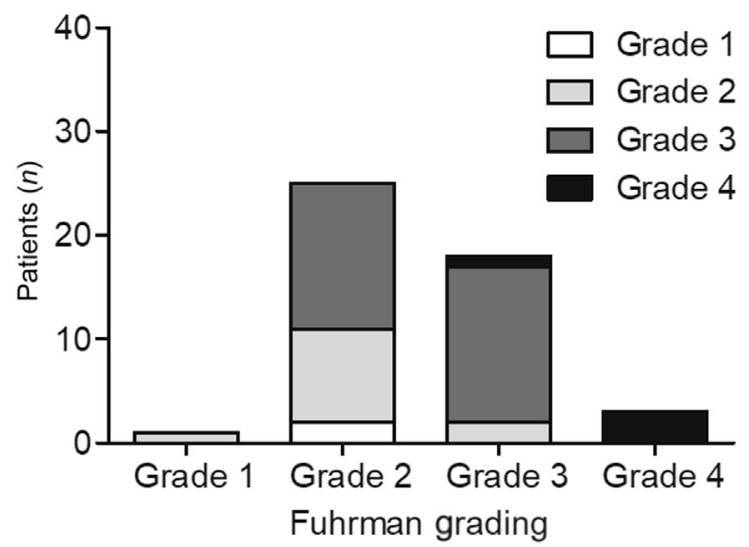

Figure 1 Distribution of World Health Organization/International Society of Urological Pathology (WHO/ISUP) grading in Fuhrman grading. 
Table 3 Morphometric Parameters for 47 Cases of Xp11.2 tRCC

\begin{tabular}{lrrl}
\hline Parameter & \multicolumn{1}{l}{ Range } & Mean & \multicolumn{1}{l}{ SD } \\
\hline Nuclear size & & & \\
$\quad$ Nuclear area, $\mu \mathrm{m}^{2}$ & $16.60-58.70$ & 31.84 & 9.12 \\
$\quad$ Nuclear perimeter, $\mu \mathrm{m}$ & $14.70-30.10$ & 21.82 & 3.41 \\
$\quad$ Major axis length, $\mu \mathrm{m}$ & $5.30-10.20$ & 7.72 & 0.96 \\
Nuclear shape & & & \\
$\quad$ Shape factor & $0.71-0.97$ & 0.83 & 0.066 \\
$\quad$ Compactness & $12.94-17.73$ & 15.23 & 1.22 \\
\hline
\end{tabular}

tRCC, translocation renal cell carcinoma.

The ranges of the nuclear size and shape parameter for all cases are summarized in Table 3. The means and 95\% CIs of the nuclear size and nuclear shape parameters, based on the Fuhrman grading system, are shown in Table 4. Measurements of nuclear size were correlated significantly with TNM staging and pT staging, but associated insignificantly with the conventional Fuhrman grading, WHO/ISUP grading, or lymph node or distant metastasis. Parameters of nuclear shape, however, correlated with none of the earlierdescribed factors (Table 5).

Two girls were lost to follow-up evaluation after surgery. For the remaining 45 cases, the duration of follow-up evaluation ranged from 1 to 114 months, with a median time of 37 months. Until the last follow-up evaluation, 13 $(27.7 \%)$ cases were disease-free over a 5-year period. However, $13(27.7 \%)$ patients were found to have disease progression and 6 patients $(12.8 \%)$ died of tumor-related causes. The 5-year PFS and OS were $64.6 \%$ and $83.1 \%$, respectively. After removal of children ( $<18$ years) from the investigation, the 5-year PFS and OS for adult patients were $60.4 \%$ and $81.0 \%$, respectively (Figure 2).

By univariate analysis, no correlation was found between the age, sex, surgery approach, conventional/simplified two-/three-tiered Fuhrman grading, or WHO/ISUP grading with survival (either PFS or OS) (Table 6). However, tumor size, TNM staging, and all of the nuclear size-associated parameters were correlated with survival. Thus, parameters of nuclear size stand in clear contrast with parameters of nuclear shape. To our disappointment, none of the correlations reached statistical significance on multivariate analysis.

\section{Discussion}

Although the Fuhrman and WHO/ISUP grading system are both widely applied in clinical practice for the grading of renal epithelial malignancies, there is little evidence indicating that they are prognostic for tumor types other than CCRCC or PRCC. Interestingly, in this study of Xp11.2 tRCC, it was nuclear size but not Fuhrman or the WHO/ISUP that was associated significantly with survival.

Xp11.2 tRCC was first reported by de Jong et al ${ }^{12}$ in 1988 and was first listed as a specific disease entity by the WHO Classification of Tumors in 2004. ${ }^{13}$ Despite decades of discovery, the clinical and prognostic feature of Xp11.2 tRCC still was poorly recognized because of its low incidence. ${ }^{1}$ Xp11.2 tRCC tends to be more aggressive than CCRCC, ${ }^{1,2}$ the most common type of RCC among adults, which was reported to have a 5-year survival rate of $81.5 \% .^{14}$ However, if all of the cases were included, only $35.4 \%$ of patients in our investigation showed disease progression in the initial 5 years and the 5-year OS reached $83.1 \%$. Once the pediatric cases were removed, the survival curves of PFS and OS were distinctly decreased, suggesting a poorer prognosis in adults than children, which was in accordance with Ellis et al. ${ }^{2}$ In addition, Rais-Bahrami et $\mathrm{al}^{15}$ reported a boy with pT1aNxMx Xp11.2 tRCC who relapsed 17 years after surgery. The 47 cases in our study were followed up for 114 months. No progressive cases were observed after a 5 -year follow-up period.

To reflect tumor cell differentiation and heteromorphism, an ideal nuclear grading system should be able to provide prognostic information and assist in predicting tumor behavior. Because the Fuhrman grading system has no proven prognostic impact in chromophobe RCC and other subtypes, the International Union Against Cancer and the American Joint Committee on Cancer suggest the application of this grading system only for CCRCC and PRCC. ${ }^{4}$ To date, limited studies addressing Xp11.2 tRCC have reported Fuhrman grading in association with information about patients' survival and quality of life. Table 7 lists the nine latest clinical studies that address Xp11.2 tRCC, but only four of them have provided data that include Fuhrman grading and patient survival. However, none of these studies showed a correlation between Fuhrman grading and

Table 4 Measurements of Nuclear Size and Shape Based on Conventional Four-Tiered Fuhrman Grading

\begin{tabular}{lccccc}
\hline & \multicolumn{2}{c}{ Grade 2} & & Grade 3 & \\
\cline { 2 - 3 } Perimeter & Mean & $95 \%$ CI & & Mean & $95 \%$ CI \\
\hline Nuclear size & & & & \\
$\quad$ Nuclear area, $\mu \mathrm{m}^{2}$ & 28.94 & $25.35-32.52$ & 34.84 & $29.70-40.00$ \\
$\quad$ Nuclear perimeter, $\mu \mathrm{m}$ & 20.87 & $19.40-22.35$ & 22.81 & $20.94-24.67$ & 0.048 \\
$\quad$ Major axis length, $\mu \mathrm{m}$ & 7.50 & $7.12-7.87$ & 7.97 & 0.090 \\
Nuclear shape & & & & 0.150 \\
$\quad$ Shape factor & 0.83 & $0.79-0.86$ & 15.14 & $0.80-0.87$ \\
$\quad$ Compactness & 12.29 & $14.65-15.93$ & & $14.54-17.74$ \\
\hline
\end{tabular}


Table 5 Comparison of Morphometric Measurements of Nuclear Size and Shape against Conventional Fuhrman Grading, WHO/ISUP Grading, TNM Staging, pT Staging, and Lymph Node Metastasis or Metastasis Distance

\begin{tabular}{|c|c|c|c|c|c|c|c|c|c|c|}
\hline \multirow[b]{2}{*}{ Perimeter } & \multicolumn{2}{|c|}{$\begin{array}{l}\text { Conventional } \\
\text { Fuhrman grade }\end{array}$} & \multicolumn{2}{|c|}{ WH0/ISUP grade } & \multicolumn{2}{|c|}{ TNM stage } & \multicolumn{2}{|l|}{ pT stage } & \multicolumn{2}{|c|}{$\begin{array}{l}\text { Lymph node or } \\
\text { distant metastasis }\end{array}$} \\
\hline & $F$ value & $P$ value & $F$ value & $P$ value & $F$ value & $P$ value & $F$ value & $P$ value & $F$ value & $P$ value \\
\hline Nuclear area & 2.580 & 0.070 & 2.833 & 0.053 & 4.448 & 0.010 & 3.646 & 0.022 & 3.495 & 0.070 \\
\hline Nuclear perimeter & 1.748 & 0.176 & 2.194 & 0.107 & 4.505 & 0.009 & 3.699 & 0.021 & 2.738 & 0.107 \\
\hline Major axis length & 1.299 & 0.291 & 1.400 & 0.260 & 3.085 & 0.040 & 3.067 & 0.041 & 1.210 & 0.279 \\
\hline Compactness & 0.081 & 0.970 & 0.835 & 0.484 & 1.179 & 0.332 & 1.347 & 0.276 & 0.004 & 0.949 \\
\hline
\end{tabular}

PT, pathological tumor invasion stage; TNM, tumor node metastasis; tRCC, translocation renal cell carcinoma; WHO/ISUP, World Health Organization/International Society of Urological Pathology.

survival. Our study, for the first time, systematically evaluated the Fuhrman grading system and provides compelling data showing that neither conventional four-tiered Fuhrman grading nor simplified two- or three-tiered Fuhrman grading is meaningful in predicting survival of Xp11.2 tRCC.

As a newly proposed nuclear grading system, the WHO/ ISUP grading system has been widely accepted. ${ }^{7}$ WHO/ISUP grading prioritizes one of the components of the Fuhrman grading: nucleolar prominence. Distinct from the first three categories of the Fuhrman grading system, which is based on the subjective assessment of three separate features, the first three grades in the WHO/ISUP grading system use more objective and straightforward criteria. Furthermore, WHO/ ISUP grading is no less valid in predicting the prognosis, particularly for CCRCC and PRCC. ${ }^{24,25}$ To explore the relevance of the WHO/ISUP grading system in Xp11.2 tRCC patients, Qu et $\mathrm{al}^{26}$ reported 36 adults who were diagnosed by fluorescence in situ hybridization. In that study, advanced WHO/ISUP grading was correlated significantly with poorer OS by univariate analysis. However, the correlation disappeared when WHO/ISUP grading was modeled with TNM staging and tumor size analysis by multivariate analyses. In our study, neither PFS nor OS was shown to be correlated with prognosis by either univariate or multivariate analysis.

Fuhrman grading assumes the same weight to nucleolar prominence, nuclear size, and nuclear shape in predicting

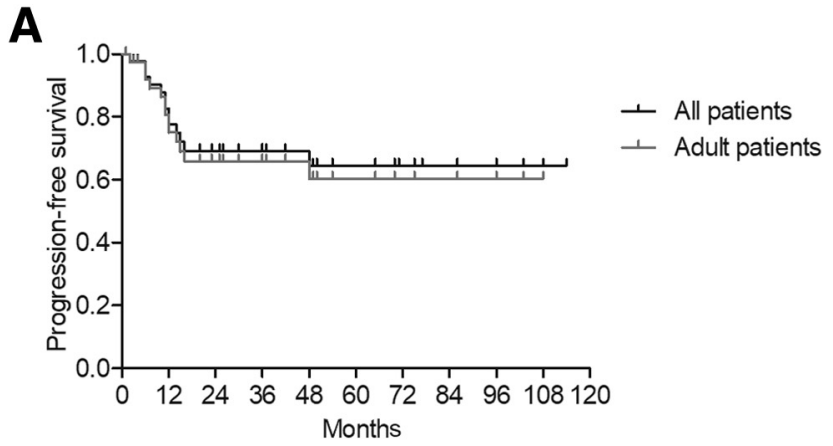

prognosis. In our investigation, an obvious discordance between WHO/ISUP grading and measurements of nuclear size and shape was recorded, which resulted in inconsistent Fuhrman grading conclusions by the pathologist objectively. Although some pathologists prefer to rely on nucleolar prominence alone when grading renal tumors, our data show that WHO/ISUP grading is not reliable as a prognostic indicator. Personal preference and selection uncertainty may account for our conclusion that Fuhrman grading is not reliable.

To our surprise, it is the nuclear size that showed a statistically significant correlation with survival on univariate testing, albeit the significance was lost on multivariate analysis. These results were consistent with the study of CCRCC by Delahunt et al. ${ }^{25}$ Nevertheless, in our Cox proportionate hazards model, the parameters of nuclear size were continuous data rather than categoric data, thus more Xp11.2 tRCC patients need to be recruited to determine the grading quantitatively. Furthermore, the major axis of a Xp11.2 tRCC tumor cell was smaller than what is defined in the Fuhrman classification for grades 1 to 3 tumors. This finding is consistent with some other types of RCC. ${ }^{25,27}$ Of note, diversified architectures of Xp11.2 tRCC and a lack of justifiable criteria for assessing nuclear shape might be responsible for the poor correlation between nuclear shape and survival.

Apart from conventional prognostic factors such as tumor grading and TNM staging, several other potential prognostic

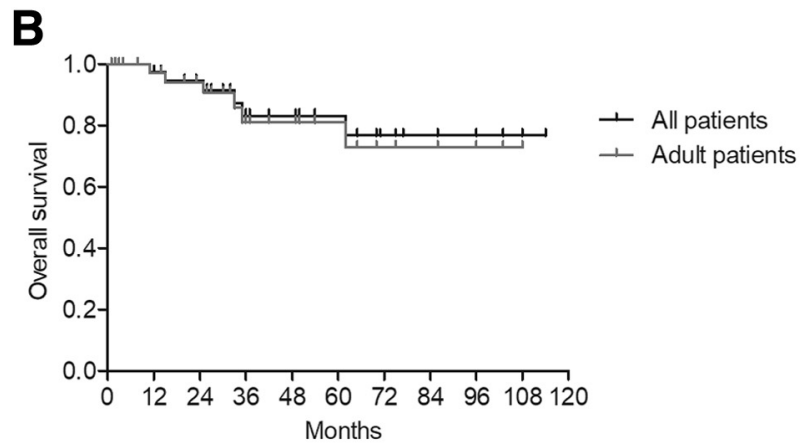

Figure 2 Kaplan-Meier survival curves of all patients and adult patients ( $\geq 18$ years). A: Progression-free survival of all patients and adult patients. B: Overall survival of all patients and adult patients. 
Table 6 Univariate-Cox Regression Analysis of PFS and OS in Xp11.2 tRCC Patients

\begin{tabular}{|c|c|c|c|c|}
\hline \multirow[b]{2}{*}{ Parameters } & \multicolumn{2}{|l|}{ PFS } & \multicolumn{2}{|l|}{ OS } \\
\hline & $\mathrm{HR}(95 \% \mathrm{CI})$ & $P$ value & $\mathrm{HR}(95 \% \mathrm{CI})$ & $P$ value \\
\hline Age & $1.039(0.993-1.088)$ & 0.099 & $1.062(0.981-1.150)$ & 0.138 \\
\hline Tumor size & $1.354(1.111-1.651)$ & 0.003 & $1.362(0.982-1.899)$ & 0.064 \\
\hline Surgery, radical/partical & $3.059(0.380-24.601)$ & 0.293 & $27.944(0-1709061.499)$ & 0.554 \\
\hline TNM stage & NA & 0.020 & NA & 0.916 \\
\hline IV/I & NA & 0.991 & $0.974(0-8.826 \times 1038)$ & 1.000 \\
\hline Conventional Fuhrman grade & NA & 0.722 & NA & 0.257 \\
\hline $\mathrm{II} / \mathrm{I}$ & $3879.537\left(0-7.491 \times 10^{113}\right)$ & 0.949 & $0.157(0.014-1.773)$ & 0.134 \\
\hline III/I & $3091.052\left(0-5.974 \times 10^{113}\right)$ & 0.951 & $0.128(0.008-2.122)$ & 0.151 \\
\hline IV/I & $11624.287\left(0-2.259 \times 10^{114}\right)$ & 0.942 & NA & 0.257 \\
\hline Simplified two-tiered Fuhrman grade (III + IV/I + II) & $1.023(0.275-3.812)$ & 0.973 & $1.453(0.204-10.343)$ & 0.709 \\
\hline Simplified three-tiered Fuhrman grade & NA & 0.511 & NA & 0.257 \\
\hline $\mathrm{III} / \mathrm{I}+\mathrm{II}$ & $0.834(0.199-3.495)$ & 0.804 & $0.813(0.073-9.006)$ & 0.866 \\
\hline IV/I + II & $3.126(0.326-26.956)$ & 0.300 & $6.357(0.564-71.644)$ & 0.134 \\
\hline Nuclear size & NA & & NA & NA \\
\hline Area & $1.097(1.030-1.168)$ & 0.004 & $1.142(1.029-1.266)$ & 0.012 \\
\hline Perimeter & $1.325(1.075-1.634)$ & 0.008 & $1.643(1.107-2.440)$ & 0.014 \\
\hline Major axis & $2.745(1.341-5.618)$ & 0.006 & $3.517(1.130-10.943)$ & 0.030 \\
\hline Nuclear shape & NA & & NA & NA \\
\hline Shape factor & $0.136(0-3361.980)$ & 0.699 & $0.001(0-4591.206)$ & 0.376 \\
\hline Compactness & $1.115(0.647-1.923)$ & 0.695 & $1.461(0.659-3.241)$ & 0.351 \\
\hline
\end{tabular}

HR, hazard ratio; NA, not available; OS, overall survival; PFS, progression-free survival; TNM, tumor node metastasis; WHO/ISUP, World Health Organization/ International Society of Urological Pathology.

parameters are worth attention. The psammoma body is a basophilic structure with laminated concretions whose diameter generally ranges from 20 to $100 \mu \mathrm{m} .{ }^{28}$ In fact, psammoma bodies are composed of calcium apatite and are first observed in meningiomas. ${ }^{29}$ Derived from degeneration of tumor cells and for suppressing the growth of neoplasms, the formation of the psammoma body, in turn, can serve as a barrier against the spread of neoplasms. ${ }^{30}$ It is known that the Xp11.2 tRCC with the ASPL-TFE3 gene fusion have more psammoma bodies than those with the PRCC-TFE3
RCC; however, the cases with the ASPL-TFE3 RCC more frequently present at an advanced stage. ${ }^{31}$ The validity of the psammoma body in predicting the prognosis of Xp11.2 tRCC is in progress in our center. Another prognostic parameter proposed by the ISUP Consensus Conference in 2012 is tumor necrosis. The predicting role of tumor necrosis for CCRCC has been identified, but for other RCC morphotypes it still is controversial. To reach a consensus, the conference proposed that the assessment of necrosis should be based on macroscopic and microscopic

Table 7 Distribution of Fuhrman Grades for the Recruited Xp11.2 tRCC and the Correlation between Fuhrman and Survival

\begin{tabular}{|c|c|c|c|c|}
\hline Study & Patients, $n$ & Diagnosis methods & Date available & $\overline{\text { Significant }}$ \\
\hline Camparo et $\mathrm{al}^{16}$ & 29 & CK/IHC & Yes & No \\
\hline Choueiri et $\mathrm{al}^{17}$ & 15 & CK/FISH/IHC & No & - \\
\hline Rao et $\mathrm{al}^{18}$ & 19 & IHC & Yes & No \\
\hline Hung et $\mathrm{al}^{19}$ & 8 & IHC & No & - \\
\hline Macher-Goeppinger ${ }^{20}$ & 8 & PCR/FISH/IHC & No & - \\
\hline Sukov et $\mathrm{al}^{1}$ & 6 & FISH & No & - \\
\hline Su et $\mathrm{al}^{21}$ & 8 & IHC & Yes & No \\
\hline Lim et $\mathrm{al}^{22}$ & 21 & $\mathrm{IHC}$ & Yes & No \\
\hline Qu et $\mathrm{al}^{23}$ & 30 & IHC/FISH & No & - \\
\hline
\end{tabular}

CK, cytogenetic karyotype; FISH, fluorescence in situ hybridization; IHC, immunohistochemical; tRCC, translocation renal cell carcinoma. 
examination using a quantitative approach. In our study, macroscopic assessment and the quantitative estimation of tumor necrosis were not possible because of the shortage of complete tumor samples. Further investigation should focus on the correlation between tumor necrosis and survival in Xp11.2 tRCC patients.

\section{Limitations}

There were certain limitations in this study. On one hand, this was a retrospective study and the sample size of Xp11.2 tRCC was insufficient because of its peculiarity, although this was one of the largest single-center clinical reports on Xp11.2 tRCC. On the other hand, no central pathologic review was performed. Nevertheless, all specimens in our study were reviewed by two experienced uropathologists and the risk of interobserver variability appears comparatively low. Notwithstanding these limitations, results from the relatively large-scale study underscored that neither the Fuhrman nor the WHO/ISUP grading system was applicable for Xp11.2 tRCC.

\section{Conclusions}

This study explored the role of two types of the most common grading system in predicting survival by studying a series of Xp11.2 tRCC patients in our center in the past decade. Both Fuhrman and WHO/ISUP grading, including conventional and simplified Fuhrman grading systems, showed poor correlation with survival. Evolved from nucleolar prominence assessment, WHO/ISUP grading shows discordance between nuclear size and nuclear shape. Somewhat surprisingly, it is the nuclear size rather than nucleolar prominence that is suggested to be a valid predictor of survival. Future studies with a larger sample size are warranted to confirm our findings.

\section{Acknowledgments}

We thank Xiaogong Li, Gutian Zhang, Linfang Yao, and Xiaozhi Zhao (Nanjing Drum Tower Hospital) for providing patient information, and Jun Yang and Ming Chen (Nanjing Drum Tower Hospital) for providing technical assistance.

\section{Supplemental Data}

Supplemental material for this article can be found at https://doi.org/10.1016/j.ajpath.2017.12.018.

\section{References}

1. Sukov WR, Hodge JC, Lohse CM, Leibovich BC, Thompson RH, Pearce KE, Wiktor AE, Cheville JC: TFE3 rearrangements in adult renal cell carcinoma: clinical and pathologic features with outcome in a large series of consecutively treated patients. Am J Surg Pathol 2012 36:663-670

2. Ellis CL, Eble JN, Subhawong AP, Martignoni G, Zhong M, Ladanyi M, Epstein JI, Netto GJ, Argani P: Clinical heterogeneity of Xp11 translocation renal cell carcinoma: impact of fusion subtype, age, and stage. Mod Pathol 2014, 27:875-886

3. Liu N, Wang Z, Gan W, Xiong L, Miao B, Chen X, Guo H, Li D: Renal cell carcinoma associated with Xp11.2 translocation/TFE3 gene fusions: clinical features, treatments and prognosis. PLoS One 2016, 11:e0166897

4. Medeiros LJ, Jones EC, Aizawa S, Aldape HC, Cheville JC, Goldstein NS, Lubensky IA, Ro J, Shanks J, Pacelli A, Jung SH: Grading of renal cell carcinoma: Workgroup No. 2. Union Internationale Contre le Cancer and the American Joint Committee on Cancer (AJCC). Cancer 1997, 80:990-991

5. Fuhrman SA, Lasky LC, Limas C: Prognostic significance of morphologic parameters in renal cell carcinoma. Am J Surg Pathol $1982,6: 655-663$

6. Al-Aynati M, Chen V, Salama S, Shuhaibar H, Treleaven D, Vincic L: Interobserver and intraobserver variability using the Fuhrman grading system for renal cell carcinoma. Arch Pathol Lab Med 2003, 127:593-596

7. Moch H, Cubilla AL, Humphrey PA, Reuter VE, Ulbright TM: The 2016 WHO classification of tumours of the urinary system and male genital organs-part A: renal, penile, and testicular tumours. Eur Urol 2016, 70:93-105

8. Delahunt B, Cheville JC, Martignoni G, Humphrey PA, MagiGalluzzi C, McKenney J, Egevad L, Algaba F, Moch H, Grignon DJ, Montironi R, Srigley JR; Members of the ISUP Renal Tumor Panel: the International Society of Urological Pathology (ISUP) grading system for renal cell carcinoma and other prognostic parameters. Am J Surg Pathol 2013, 37:1490-1504

9. Zisman A, Pantuck AJ, Dorey F, Said JW, Shvarts O, Quintana D, Gitlitz BJ, deKernion JB, Figlin RA, Belldegrun AS: Improved prognostication of renal cell carcinoma using an integrated staging system. J Clin Oncol 2001, 19:1649-1657

10. Ficarra V, Martignoni G, Maffei N, Brunelli M, Novara G, Zanolla L, Pea M, Artibani W: Original and reviewed nuclear grading according to the Fuhrman system: a multivariate analysis of 388 patients with conventional renal cell carcinoma. Cancer 2005, 103:68-75

11. Chen X, Yang Y, Gan W, Xu L, Ye Q, Guo H: Newly designed breakapart and ASPL-TFE3 dual-fusion FISH assay are useful in diagnosing Xp11.2 translocation renal cell carcinoma and ASPL-TFE3 renal cell carcinoma: a STARD-compliant article. Medicine (Baltimore) 2015, 94:e873

12. de Jong B, Oosterhuis JW, Idenburg VJ, Castedo SM, Dam A, Mensink HJ: Cytogenetics of 12 cases of renal adenocarcinoma. Cancer Genet Cytogenet 1988, 30:53-61

13. Lopez-Beltran A, Scarpelli M, Montironi R, Kirkali Z: 2004 WHO classification of the renal tumors of the adults. Eur Urol 2006, 49: 798-805

14. Sun M, Lughezzani G, Jeldres C, Isbarn H, Shariat SF, Arjane P, Widmer H, Pharand D, Latour M, Perrotte P, Patard JJ, Karakiewicz PI A proposal for reclassification of the Fuhrman grading system in patients with clear cell renal cell carcinoma. Eur Urol 2009, 56:775-781

15. Rais-Bahrami S, Drabick JJ, De Marzo AM, Hicks J, Ho C, Caroe AE, Argani P: Xp11 translocation renal cell carcinoma: delayed but massive and lethal metastases of a chemotherapy-associated secondary malignancy. Urology 2007, 70:178.e3-178.e6

16. Camparo P, Vasiliu V, Molinie V, Couturier J, Dykema KJ, Petillo D, Furge KA, Comperat EM, Lae M, Bouvier R, Boccon-Gibod L, Denoux Y, Ferlicot S, Forest E, Fromont G, Hintzy MC, Laghouati M, Sibony M, Tucker ML, Weber N, Teh BT, Vieillefond A: Renal translocation carcinomas: clinicopathologic, immunohistochemical, and gene expression profiling analysis of 31 cases with a review of the literature. Am J Surg Pathol 2008, 32:656-670

17. Choueiri TK, Lim ZD, Hirsch MS, Tamboli P, Jonasch E, McDermott DF, Dal Cin P, Corn P, Vaishampayan U, Heng DY, 
Tannir NM: Vascular endothelial growth factor-targeted therapy for the treatment of adult metastatic Xp11.2 translocation renal cell carcinoma. Cancer 2010, 116:5219-5225

18. Rao Q, Chen JY, Wang JD, Ma HH, Zhou HB, Lu ZF, Zhou XJ: Renal cell carcinoma in children and young adults: clinicopathological, immunohistochemical, and VHL gene analysis of 46 cases with follow-up. Int J Surg Pathol 2011, 19:170-179

19. Hung CC, Pan CC, Lin CC, Lin AT, Chen KK, Chang YH: XP11.2 translocation renal cell carcinoma: clinical experience of Taipei Veterans General Hospital. J Chin Med Assoc 2011, 74:500-504

20. Macher-Goeppinger S, Roth W, Wagener N, Hohenfellner M, Penzel R, Haferkamp A, Schirmacher P, Aulmann S: Molecular heterogeneity of TFE3 activation in renal cell carcinomas. Mod Pathol 2012, 25:308-315

21. Su HH, Sung MT, Chiang PH, Cheng YT, Chen YT: The preliminary experiences of translocation renal cell carcinoma and literature review. Kaohsiung J Med Sci 2014, 30:402-408

22. Lim B, You D, Jeong IG, Kwon T, Hong S, Song C, Cho YM, Hong B, Hong JH, Ahn H, Kim CS: Clinicopathological features of Xp11.2 translocation renal cell carcinoma. Korean J Urol 2015, 56:212-217

23. Qu Y, Gu C, Wang H, Chang K, Yang X, Zhou X, Dai B, Zhu Y, Shi G, Zhang H, Ye D: Diagnosis of adults Xp11.2 translocation renal cell carcinoma by immunohistochemistry and FISH assays: clinicopathological data from ethnic Chinese population. Sci Rep 2016, 6:21677

24. Sika-Paotonu D, Bethwaite PB, McCredie MR, William Jordan T, Delahunt B: Nucleolar grade but not Fuhrman grade is applicable to papillary renal cell carcinoma. Am J Surg Pathol 2006, 30:1091-1096
25. Delahunt B, Sika-Paotonu D, Bethwaite PB, William Jordan T, MagiGalluzzi C, Zhou M, Samaratunga H, Srigley JR: Grading of clear cell renal cell carcinoma should be based on nucleolar prominence. Am J Surg Pathol 2011, 35:1134-1139

26. Qu Y, Zhao R, Wang H, Chang K, Yang X, Zhou X, Dai B, Zhu Y, Shi G, Zhang H, Ye D: Phosphorylated 4EBP1 is associated with tumor progression and poor prognosis in Xp11.2 translocation renal cell carcinoma. Sci Rep 2016, 6:23594

27. Delahunt B, Sika-Paotonu D, Bethwaite PB, McCredie MR, Martignoni G, Eble JN, Jordan TW: Fuhrman grading is not appropriate for chromophobe renal cell carcinoma. Am J Surg Pathol 2007, 31:957-960

28. Fadare O, Chacho MS, Parkash V: Psammoma bodies in cervicovaginal smears: significance and practical implications for diagnostic cytopathology. Adv Anat Pathol 2004, 11:250-261

29. Kubota T, Hirano A, Sato K, Yamamoto S: Fine structure of psammoma bodies at the outer aspect of blood vessels in meningioma. Acta Neuropathol 1985, 66:163-166

30. Das DK: Psammoma body: a product of dystrophic calcification or of a biologically active process that aims at limiting the growth and spread of tumor? Diagn Cytopathol 2009, 37:534-541

31. Argani P, Antonescu CR, Illei PB, Lui MY, Timmons CF, Newbury R, Reuter VE, Garvin AJ, Perez-Atayde AR, Fletcher JA, Beckwith JB, Bridge JA, Ladanyi M: Primary renal neoplasms with the ASPL-TFE3 gene fusion of alveolar soft part sarcoma: a distinctive tumor entity previously included among renal cell carcinomas of children and adolescents. Am J Pathol 2001, 159:179-192 